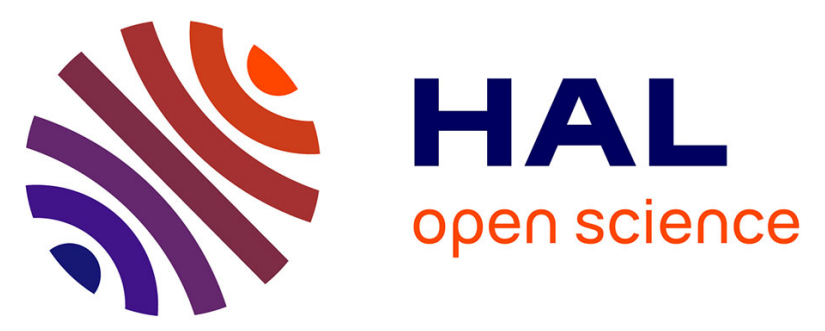

\title{
Uncertainty quantification for an industrial mistuned bladed disk with geometrical nonlinearities
}

\author{
Evangéline Capiez-Lernout, Christian Soize, Moustapha Mbaye
}

\section{To cite this version:}

Evangéline Capiez-Lernout, Christian Soize, Moustapha Mbaye. Uncertainty quantification for an industrial mistuned bladed disk with geometrical nonlinearities. 60th ASME Turbo Expo 2015: Turbine Technical Conference and Exposition, ASME, Jun 2015, Montreal, Canada. pp.1-10. hal-01164483

\section{HAL Id: hal-01164483 \\ https://hal.science/hal-01164483}

Submitted on 17 Jun 2015

HAL is a multi-disciplinary open access archive for the deposit and dissemination of scientific research documents, whether they are published or not. The documents may come from teaching and research institutions in France or abroad, or from public or private research centers.
L'archive ouverte pluridisciplinaire HAL, est destinée au dépôt et à la diffusion de documents scientifiques de niveau recherche, publiés ou non, émanant des établissements d'enseignement et de recherche français ou étrangers, des laboratoires publics ou privés. 
ASME Turbo Expo 2015, June 15-19, 2015, Montréal, Canada

\section{UNCERTAINTY QUANTIFICATION FOR AN INDUSTRIAL MISTUNED BLADED DISK WITH GEOMETRICAL NONLINEARITIES.}

\author{
Evangéline Capiez-Lernout \\ Université Paris-Est \\ Modélisation et Simulation \\ Multi-Echelle \\ MSME UMR 8208 CNRS \\ 77454 Marne-la-Vallée Cedex 02 \\ France \\ evangeline.capiez-lernout@u-pem.fr
}

\author{
Christian Soize \\ Université Paris-Est \\ Modélisation et Simulation \\ Multi-Echelle \\ MSME UMR 8208 CNRS \\ 77454 Marne-la-Vallée Cedex 02 \\ France \\ christian.soize@u-pem.fr
}

\author{
Moustapha Mbaye \\ Turbomeca - Safran Group \\ 64511 Bordes, France \\ moustapha.mbaye@turbomeca.fr
}

\begin{abstract}
Recently, a methodology allowing a stochastic nonlinear reduced-order model to be constructed in the context of the nonlinear mistuning induced by geometric nonlinearities has been proposed. The present work is devoted to an industrial application for which the centrifugal stiffening due to rotational effects is also included. The nonlinear mistuned forced response is investigated in the time-domain by using a spatial cyclic load over a given excitation frequency range. The geometric nonlinear mistuned analysis is performed over the frequency range by using the Fast-Fourier Transform of the time response. A sensitivity analysis is conducted with respect to the load level, giving rise to secondary resonances, which appear outside the excitation frequency range and which can exhibit a particular sensitivity to uncertainties. Such new complex dynamical situation, induced by the coupling between the geometrical nonlinearities and the mistuning phenomenon, is analyzed in details.
\end{abstract}

\section{NOMENCLATURE}

$b_{w, \infty}$ Amplitude ratio

$b_{v, \infty} \quad$ Frequency ratio

$f_{0} \quad$ Load level

$g(t) \quad$ Time domain of the load

$\hat{g}(2 \pi v) \quad$ Frequency domain of the load

$j_{0}, k_{0} \quad$ Blade number

$t / t_{0} \quad$ Dimensionless time

$v(t) \quad$ Displacement vector in the time domain $w(2 \pi v) \quad$ Frequency domain observation (mean NL-ROM)

$P$ Dimension of the NL-ROM

$W(2 \pi v) \quad$ Frequency domain observation (stochastic NL-ROM)

$Y(2 \pi v) \quad$ Amplification factor (stochastic NL-ROM)

$\delta$ Dispersion parameter

$v / v_{0} \quad$ Dimensionless frequency

$\Omega$ Angular speed (rotational motion)

$B$ Frequency band of analysis

$\mathbb{B}_{e}^{1}, \mathbb{B}_{e}^{2} \quad$ Frequency band of excitation

$\mathbb{B}_{s}, \mathbb{B}_{\text {sub }} \quad$ Sub-frequency range

a Spatial discretization of the load

$\mathbf{f}(t) \quad$ External load vector

$\mathbf{u}(t) \quad$ Displacement vector in the time domain

$\mathbf{u}(t) \quad$ Displacement vector in the time domain

$\widehat{\widetilde{\mathbf{u}}}(2 \pi v) \quad$ Fourier transform of the displacement vector in the frequency domain

\section{INTRODUCTION}

In general, the natural cyclic symmetry of turbomachinery bladed disks is broken because of manufacturing tolerances and material dispersions, which create small variations from one blade to another one. Such phenomena, referred to mistuning, can generate localization effects combined to a dynamic amplification of the forced response [1]. Many research efforts have been carried out on this subject, including reduced-order models with probabilistic approaches in the numerical modeling, for taking into account the random character of mistuning [2-4] 
and giving rise to strategies for the robust design of such structures [5-8]. Another essential aspect is to pay a particular attention to the various nonlinear effects that have to be taken into account in the computational models. Various research efforts have been made for the modeling of local nonlinearities such as nonlinear contact interfaces $[9,10]$ or nonlinear material damping [11]. Moreover, considering the constant growing of the computational capabilities, including the possibility of using parallel computations, it seems appropriate to consider the geometrically nonlinear effects in the computational models $[12,13]$, that can occur when exceptional operating speeds of bladed disks are analyzed due to geometric nonlinearities induced by large deformations and large displacements $[14,15]$. The recent improvements in turbomachinery design requires the analysis of exceptional operating regime of bladed disks for which large displacements/deformations can occur. The case of severe loading is investigated in the context of elastodynamics. Such situation is equivalent to nearly unstable cases induced by aerodynamic coupling yielding flutter and thus very low damping levels. In such case, the linearized elasto-dynamic theory can not be used anymore because the geometrically nonlinear effects induced by the large deformations and the large displacements are very strong and need to be taken into account in the modeling.

Recently, a methodology has been proposed for the geometric nonlinear analysis of mistuned bladed disks [16], for which all the terms including the rotational motion of the bladed-disk are taken into account. The main steps of such methodology are briefly summarized below in the context of three dimensional solid finite elements with 8 nodes.

1. Construction of the finite element model for the linear tuned case.

2. Solving the linear eigenvalue problem of the tuned structure for choosing the excitation frequency band known to yield drastic mistuning effects [1].

3. Construction of the external load in the time domain corresponding to a uniform sweep of the frequency band of excitation $[16,17]$

4. Choosing the projection basis as the linear eigenmodes of the tuned structure [18]

5. Direct construction of the nonlinear reduced-order model (NL-ROM) by projection of the finite element operators on the chosen projection basis $[19,20]$

6. Implementing the mistuning through the nonparametric probabilistic framework [21] and controlling the mistuning level by dispersion parameter $\delta=\left(\delta_{M}, \delta_{D}, \delta_{C}, \delta_{K_{c}}, \delta_{K}\right)$, each scalar controlling the dispersion of the random mass, damping, gyroscopic coupling, centrifugal stiffness, elastic stiffness matrices.

7. Solving the stochastic nonlinear equations in the timedomain with efficient algorithms based on the arc-length methods $[16,20]$
The present manuscript is devoted to an industrial application of such methodology, in order to not only demonstrate the capability of the methodology for numerical models with large number of dofs, but also to bring out a high complex dynamical behavior. In the present case, the coupling between the geometric nonlinearities and between the presence of uncertainties induced by the mistuning, give rise to new complex situations, which can be potentially dangerous for the predictions of life duration of bladed-disks structures, and which have to be carefully analyzed.

The manuscript is organized as follows: in the first section, the industrial numerical model of the bladed-disk is described. Its tuned linear dynamic analysis is used for the construction of two external loads, corresponding to situations known to be slightly and strongly sensitive to the mistuning. The second part is devoted to the nonlinear tuned dynamic analysis, for which both time domain and frequency domain are investigated. In particular, a sensitivity analysis is performed with respect to the load level in order to quantify the effects of the geometric nonlinearities. Then, a nonlinear sensitivity analysis with respect to the different classes of uncertainties induced by the mistuning is carried out in order to qualify the different mistuning effects that can occur in presence of geometric nonlinearities. Finally, a complete nonlinear dynamical analysis of the mistuned response is carried out and the confidence region of the response amplifications are compared between the linear and nonlinear cases on the whole frequency band of analysis.

\section{DESCRIPTION OF THE INDUSTRIAL APPLICATION Description of the structure}

The structure under consideration is an industrial centrifugal compressor belonging to the class of integrated bladed disks. Due to confidentiality reasons, the number of blades characterizing the order of the cyclic symmetry of the structure is not given. The finite element model of the structure is constructed with solid finite elements and is constituted of about 2,000,000 degrees of freedom. Figure. 1 displays a part of the finite element mesh of the investigated bladed disk. The structure is in rotation around its revolution axis with a constant velocity $\Omega=30,750 \mathrm{rpm}$. Since the dynamic analysis is carried out in the rotating frame of the structure, the rigid body motion due to the rotation of the structure corresponds to a fixed boundary condition at the inner radius of the structure. The bladed disk is made up of a homogeneous isotropic material. A constant modal damping model is added for the bladed disk.

\section{Choice of the external load}

The cyclic symmetry is first used for constructing the reduced matrices of the mean linear reduced-order model (mean L-ROM). The linear generalized eigenvalue problem related to the tuned bladed-disk is then solved using this cyclic symmetry 


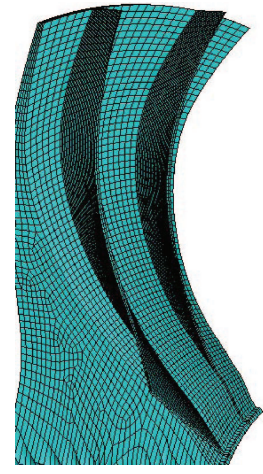

FIGURE 1. FINITE ELEMENT MESH OF A PART OF THE STRUCTURE

property. Let $v_{0}$ be the first eigenfrequency. Figure 2 displays the dimensionless eigenfrequencies $v_{i} / v_{0}$ of the tuned structure with respect to the circumferential wave number $n$. The graph is truncated to $n=5$ because only a 5 th engine-order excitation is needed to be considered in the present industrial application. Two dimensionless frequency excitation bands $\mathbb{B}_{e}^{1}=[0.97,1.10]$ and $\mathbb{B}_{e}^{2}=[1.78,2.34]$ are investigated. Frequency excitation band $\mathbb{B}_{e}^{1}$ contains an insulated resonance whereas frequency band $\mathbb{B}_{e}^{2}$ corresponds to a veering, which is known to intensify the drastic effects of linear mistuning yielding the dynamical response to be usually consequently amplified.

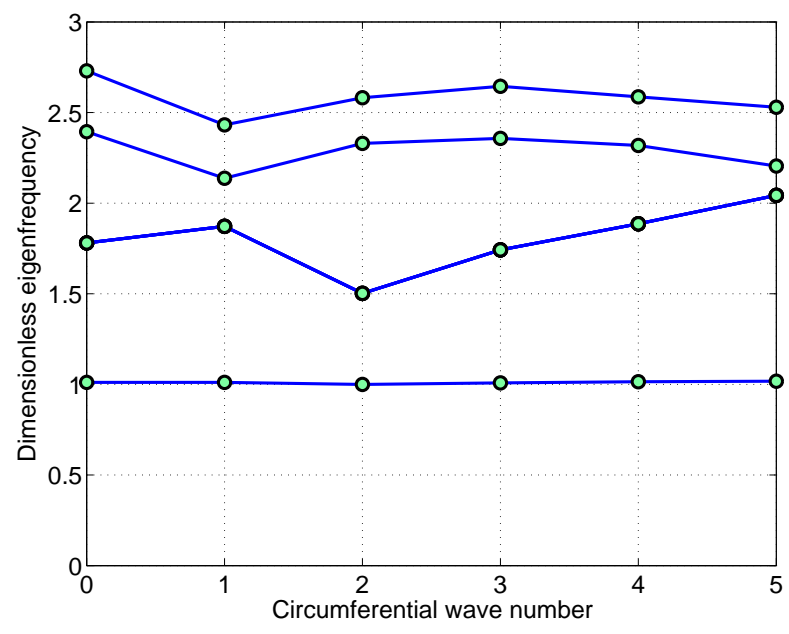

FIGURE 2. NATURAL FREQUENCIES WITH RESPECT TO CIRCUMFERENTIAL WAVE NUMBER

The external load can be written as

$$
\mathbf{f}(t)=f_{0} \mathbf{\alpha} g(t),
$$

in which the intensity of the external load $f_{0}$ is located in the range $f_{0} \in[0,6] N$, which sweeps from a negligible rate until a very high rate of geometrical nonlinearity for the numerical application presented. A high rate of loading can be interpreted as equivalent to a situation for which the damping of the bladed disk structure would reach very small values. Such extreme situations are realistic when approaching flutter regimes. In Eq. 1, the vector $\boldsymbol{\alpha}$ characterizes the spatial distribution of the load, for which point excitations are uniformly distributed at the tip of each blade along the axial direction. The chosen frequency band of excitations are described through the time domain functions $g(t)$ according to the methodology described in [17] and applied in [20]. The frequency band of analysis is a broad frequency band corresponding to the dimensionless frequency band of analysis $\mathbb{B}=[0,3.34]$. The Shannon theorem is carried out with a higher sample frequency $v_{e} / v_{0}=11.12$, yielding the number $n_{t}$ of time steps to be $n_{t}=4,096$. The frequency resolution is then $v / v_{0},=0.0054$. Let $\hat{g}(2 \pi v)$ be the Fourier transform of function $g(t)$. Figures 3 and 4 show the graphs $t / t_{0} \mapsto g(t)$ and $v / v_{0} \mapsto \hat{g}(2 \pi v)$ for both cases corresponding to excitation frequency bands $\mathbb{B}_{e}^{1}$ and $\mathbb{B}_{e}^{2}$.
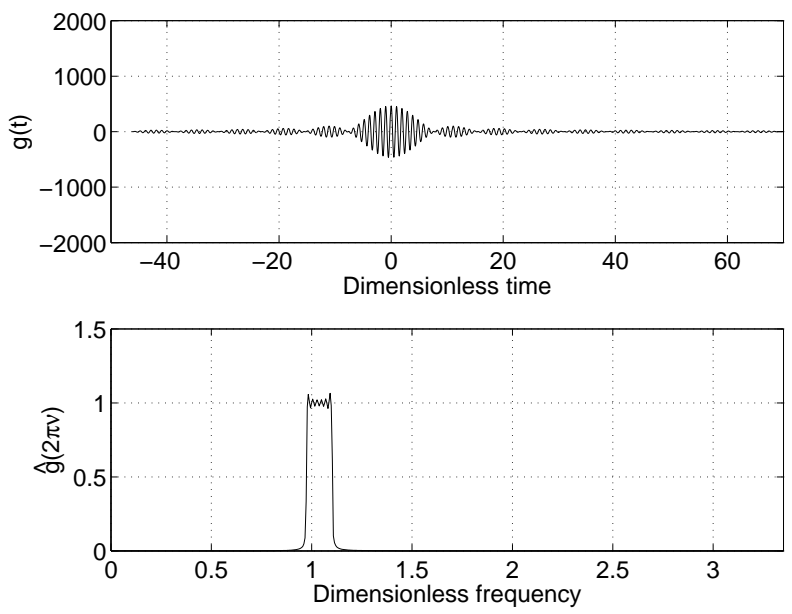

FIGURE 3. REPRESENTATION OF THE EXTERNAL LOAD IN THE TIME DOMAIN AND IN THE FREQUENCY DOMAIN: GRAPH OF $t / t_{0} \mapsto g(t)$ (UPPER GRAPH) AND $v / v_{0} \mapsto \hat{g}(2 \pi v)$ (LOWER GRAPH) FOR $\mathbb{B}_{e}^{1}=[0.97,1.10]$.

\section{NONLINEAR TUNED DYNAMIC ANALYSIS}

Concerning the choice of the vector basis for the construction of the mean NL-ROM, the nonlinear equations are solved in the subspace spanned by the usual linear basis constituted of 

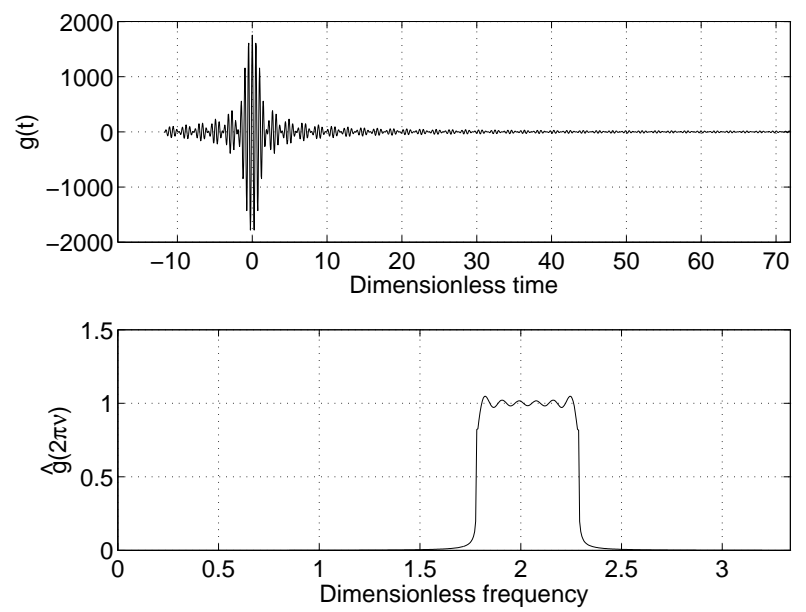

FIGURE 4. REPRESENTATION OF THE EXTERNAL LOAD IN THE TIME DOMAIN AND IN THE FREQUENCY DOMAIN: GRAPH OF $t / t_{0} \mapsto g(t)$ (UPPER GRAPH) AND $v / v_{0} \mapsto \hat{g}(2 \pi v)$ $\left(\right.$ LOWER GRAPH) FOR $\mathbb{B}_{e}^{2}=[1.78,2.34]$.

the $P$ modal shapes related to the first increasing natural eigenfrequencies, according to [19]. A convergence analysis is carried out by increasing the size $P$ of the reduced-order model. It can be shown that $P=65$ yields a reasonable convergence quantified by the $L^{2}$-norm of the frequency-domain response. From now on, the converged solution corresponding to the displacement observation from the mean NL-ROM is denoted by $\widetilde{\mathbf{u}}(t)$. For clarity, when confusion is possible, superscripts $L$ and $N L$ will be added for distinguishing the linear case from the geometric nonlinear one. The two excitation cases are investigated successively.

\section{Nonlinear tuned analysis related to excitation fre- quency band $\mathbb{B}_{e}^{1}$}

Being interested in the blade yielding the highest vibration amplitude, let $j_{0}$ be $j_{0}=\arg \max _{j}\left(\max _{t} \widetilde{u}_{j}^{N L}(t)\right)$. The observation $v(t)$ corresponding to the selected blade out-of-plane displacement is defined by $v(t)=\widetilde{u}_{j_{0}}(t)$. Figure 5 displays the graph $t / t_{0} \mapsto v^{L}(t)$ (upper graph) and $t / t_{0} \mapsto v^{N L}(t)$ (lower graph), corresponding to a load level $f_{0}=2.5 \mathrm{~N}$. Since the dynamical response related to these two cases is sensitively different, it can be deduced that $f_{0}=2.5 \mathrm{~N}$ corresponds to a load level for which the geometric nonlinear effects are significant, yielding a blade softening characterized by a relative long-term amplification of the blade displacement with respect to the linear case. There is no reason to think that the blade response shape is especially different in the frequency domain because no noticeable irregularity is observed in the time domain nonlinear response.

Let $k_{0}=\arg \max _{j}\left(\max _{v / v_{0} \in \mathbb{B}} \widehat{\widetilde{u}}_{j}^{N L}(2 \pi v)\right)$ for which
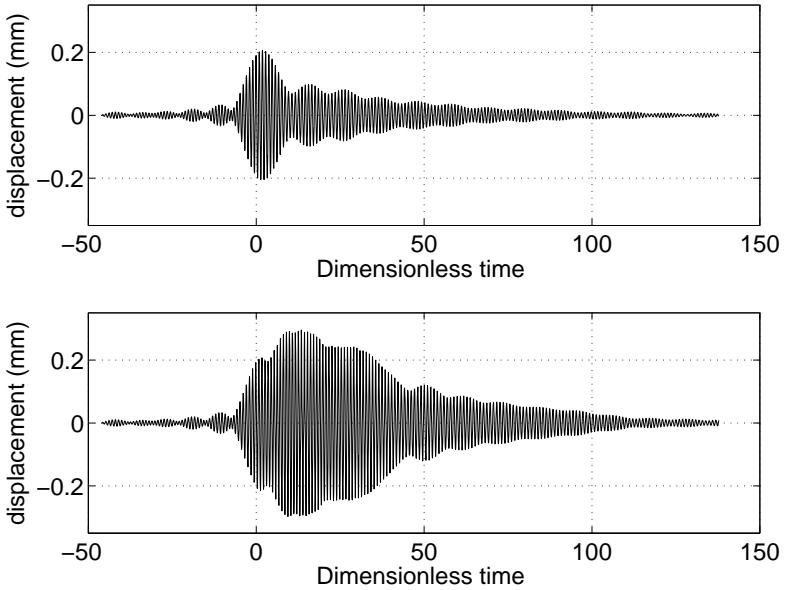

FIGURE 5. TIME DOMAIN OBSERVATION $t / t_{0} \mapsto v(t)$ RELATED TO THE LINEAR (UPPER GRAPH) AND THE NONLINEAR (LOWER GRAPH) CASES FOR $\mathbb{B}_{e}^{1}=[0.97,1.10]$ and $f_{0}=$ $2.5 N$.

$\widehat{\widetilde{u}}_{j}^{N L}(2 \pi v)$ is the Fourier transform of $\widetilde{u}_{j}^{N L}(t)$. In the frequency domain, the observation $w(2 \pi v)$ corresponding to the selected blade related to the Fourier transform of out-of-plane displacement is defined by $w(2 \pi v)=\widehat{\widetilde{u}}_{k_{0}}(2 \pi v)$. Figure 6 displays the graphs $v / v_{0} \mapsto w^{L}(2 \pi v)$ (upper graph) and $v / v_{0} \mapsto w^{N L}(2 \pi v)$ (lower graph). As expected for the linear case, it can be seen that the frequency content of the blade response coincides with $\mathbb{B}_{e}^{1}$. One can clearly see that there exist higher frequencies, which are located in dimensionless frequency band $[3,3.5]$ that are excited through the geometric nonlinearities. Nevertheless, its contribution is clearly negligible. Moreover, it is clearly seen that the dimensionless eigenfrequencies corresponding to the series of the three classes of tuned modal shapes located in dimensionless frequency band $[1.5,2.8]$ (see Fig. 2) remain insensitive to the presence of geometrical nonlinearities.

Finally, a sensitivity analysis is conducted in order to quantify the geometric nonlinear effects with respect to the load level. Let $b_{w, \infty}$ and $b_{v, \infty}$ be the amplitude ratio and frequency ratio defined by

$$
\begin{aligned}
& b_{w, \infty}=\frac{\max _{v \in \mathbb{B}} w^{N L}(2 \pi v)}{\max _{v \in \mathbb{B}} w^{L}(2 \pi v)} \\
& b_{v, \infty}=\frac{\arg \max _{v \in \mathbb{B}} w^{N L}(2 \pi v)}{\arg \max _{v \in \mathbb{B}} w^{L}(2 \pi v)} .
\end{aligned}
$$

Figure 7 displays the graphs $f_{0} \mapsto b_{w, \infty}\left(f_{0}\right)$ (upper graph) and $f_{0} \mapsto b_{v, \infty}\left(f_{0}\right)$ (lower graph). Such graphs show if the analyzed response belongs or not to the nonlinear domain of analysis. When the values of observations $b_{w, \infty}$ and $b_{v, \infty}$ are different from 
1 , the domain of analysis is nonlinear. It can be seen that geometric nonlinear effects occur from $f_{0}=0.5 \mathrm{~N}$. A non-monotonic amplification of the main resonance peak combined to a $10 \%$ shift to higher frequencies of its corresponding resonance frequency is also observed.
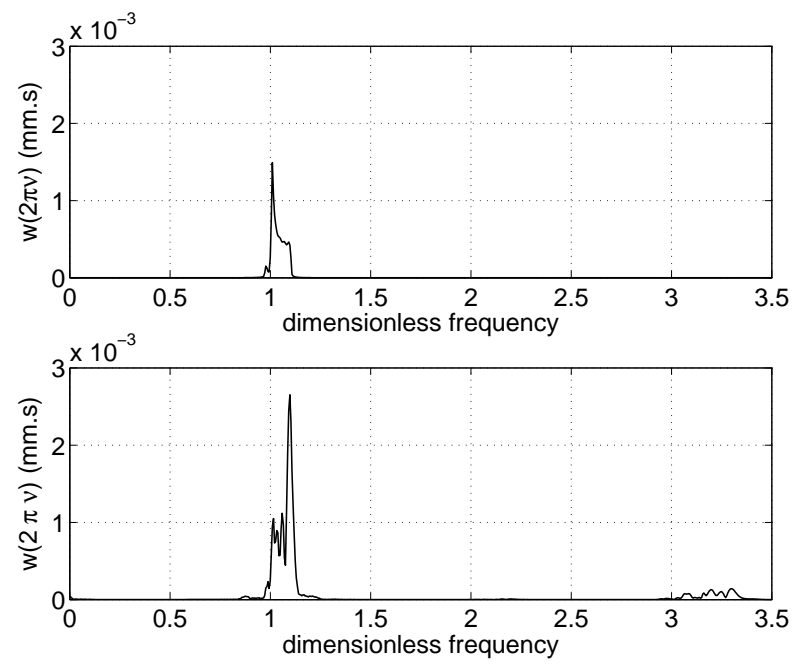

FIGURE 6. FREQUENCY DOMAIN OBSERVATION $v / v_{0} \mapsto$ $w(2 \pi v)$ RELATED TO THE LINEAR (UPPER GRAPH) AND THE NONLINEAR (LOWER GRAPH) CASES FOR $\mathbb{B}_{e}^{1}=[0.97,1.10]$ and $f_{0}=2.5 N$.
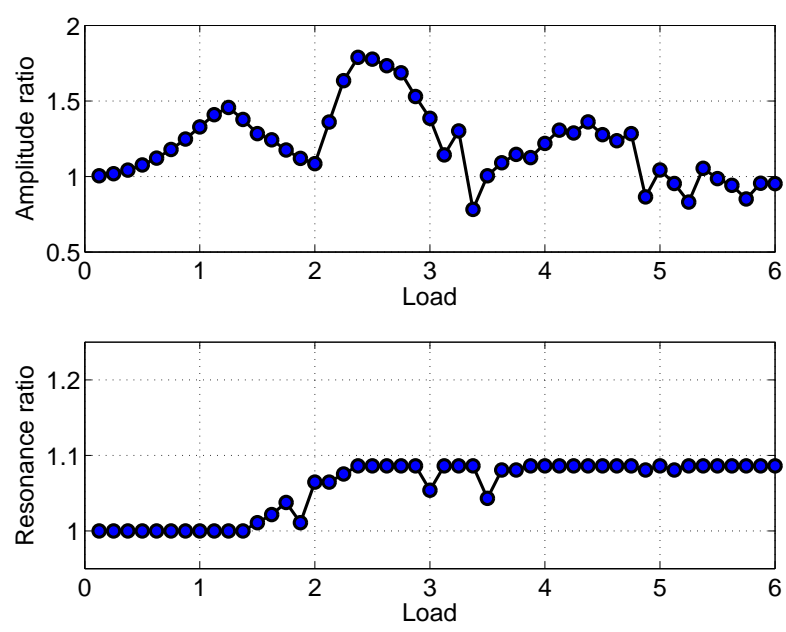

FIGURE 7. SENSITIVITY ANALYSIS WITH RESPECT TO THE LOAD LEVEL $f_{0}$.

\section{Nonlinear tuned analysis related to excitation fre- quency band $\mathbb{B}_{e}^{2}$}

Figure 8 displays the graph $t / t_{0} \mapsto v^{L}(t)$ (upper graph) and $t / t_{0} \mapsto v^{N L}(t)$ (lower graph), corresponding to a load level $f_{0}=$ $2.5 \mathrm{~N}$. On these graph, significant level of geometrical nonlinear effect can be observed and the dynamical response is drastically different from the previous case. It is seen that the geometric nonlinearities induce a blade stiffening characterized by a reduction of the vibration amplitudes of the blades from $2 \mathrm{~mm}$ until $1 \mathrm{~mm}$ with respect to the linear case. This stiffening is also combined with a strong irregularity of the blade response shape over time, which shows an enrichment of the frequency content, which has to be quantified.
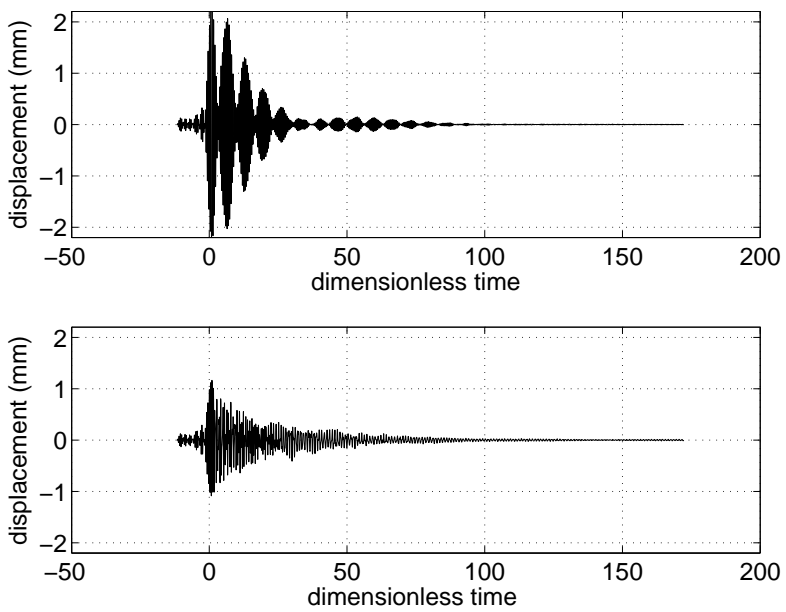

FIGURE 8. TIME DOMAIN OBSERVATION $t / t_{0} \mapsto v(t)$ RELATED TO THE LINEAR (UPPER GRAPH) AND THE NONLINEAR (LOWER GRAPH) CASES FOR $\mathbb{B}_{e}^{2}=[1.78,2.34]$ and $f_{0}=$ $2.5 N$.

Figure 9 displays the graphs $v / v_{0} \mapsto w^{L}(2 \pi v)$ (upper graph) and $v / v_{0} \mapsto w^{N L}(2 \pi v)$ (lower graph). The coupling issued from the nonlinear geometric effects is now characterized through secondary response peaks, whose frequency content covers the whole frequency band of analysis B. Again, for high frequencies, which are located outside $\mathbb{B}_{e}^{2}$ in dimensionless frequency band $[3,3.5]$, the dynamical response induced by the geometric nonlinearities remains negligible. Nevertheless, some nonnegligible contributions appears through secondary resonances with the same order of magnitude than the main resonance in the dimensionless frequency range $[1,1.5]$. One put then in evidence a complex dynamical behavior, which can be dangerous because non-expected resonances with non-negligible amplitudes appear outside the excitation frequency band. 

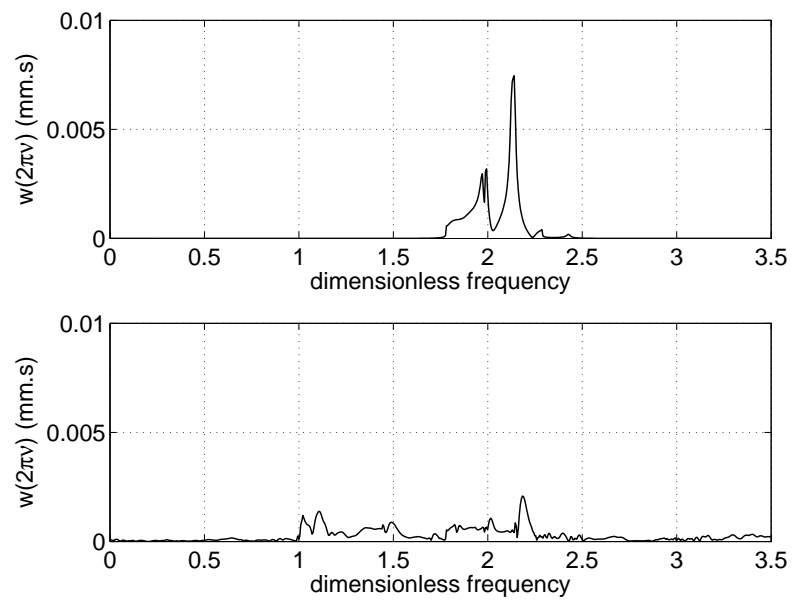

FIGURE 9. FREQUENCY DOMAIN OBSERVATION $v / v_{0} \mapsto$ $w(2 \pi v)$ RELATED TO THE LINEAR (UPPER GRAPH) AND THE NONLINEAR (LOWER GRAPH) CASES FOR $\mathbb{B}_{e}^{2}=[1.78,2.34]$ and $f_{0}=2.5 \mathrm{~N}$.

Figure 10 displays the graphs $f_{0} \mapsto b_{w, \infty}\left(f_{0}\right)$ (upper graph) and $f_{0} \mapsto b_{v, \infty}\left(f_{0}\right)$ (lower graph), which is very different from the previous case. A quick decrease in the amplitude ratio of the main resonance located in frequency band of analysis $B$ is observed with respect to the load level. More precisely, one detects that the critical load level from which the geometric nonlinearities affect the dynamical response is $f_{0}=0.375 N$. Concerning the frequency ratio, one can see a slight shift of the resonance frequency to higher frequencies for load levels lower than $f_{0}=2.5 \mathrm{~N}$. Nevertheless, a chaotic behavior is brought out from $f_{0}=2.75 \mathrm{~N}$. The resonance ratio suddenly falls down to very low values of resonance ratio belonging to $[0.5,0.8]$. A random alternating between low resonance ratios and resonance ratios slightly over 1 is shown. Such behavior means that there exist situations for which the resonances excited from the geometric nonlinearities and corresponding to subharmonics become the most important. Such observation gives rise to a worrying dynamical behavior, which points out the necessity of taking into account the geometric nonlinearities in the modeling.

Figure 11 displays the graph $t / t_{0} \mapsto v^{L}(t)$ (upper graph) and $t / t_{0} \mapsto v^{N L}(t)$ (lower graph), corresponding to a load level $f_{0}=2.75 \mathrm{~N}$. If no noticeable difference is observed in the nonlinear time domain response with respect to the case corresponding to $f_{0}=2.5 \mathrm{~N}$, this appears not to be true in the frequency domain. Figure 12 displays the graphs $v / v_{0} \mapsto w^{L}(2 \pi v)$ (upper graph) and $v / v_{0} \mapsto w^{N L}(2 \pi v)$ (lower graph). It is seen that the main resonance amplitude is nearly twice the resonance amplitude located in $\mathbb{B}_{e}^{2}$. Moreover, a broad range of frequency band $[1,1.5]$ is excited, yielding a large number of resonances with the same order magnitude than the resonance amplitude located
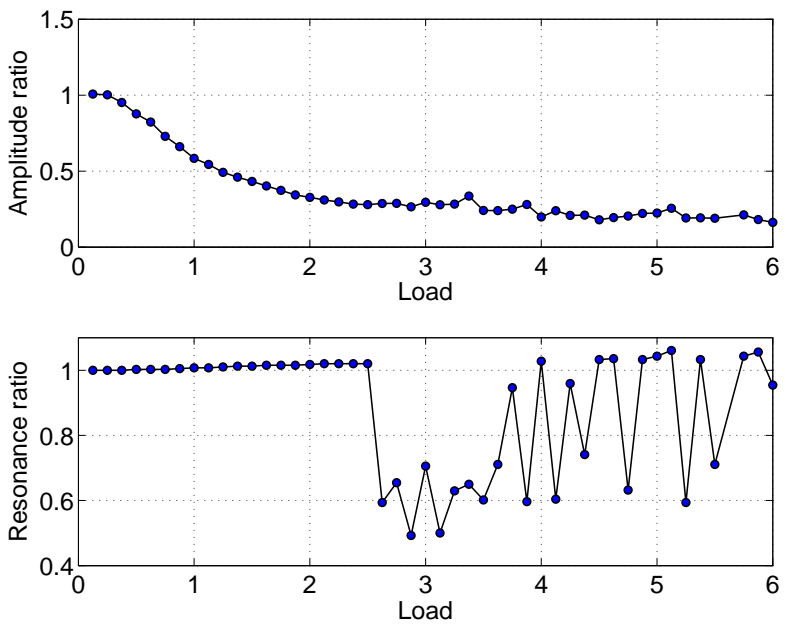

FIGURE 10. SENSITIVITY ANALYSIS WITH RESPECT TO THE LOAD LEVEL $f_{0}$.

in $\mathbb{B}_{e}^{2}$.
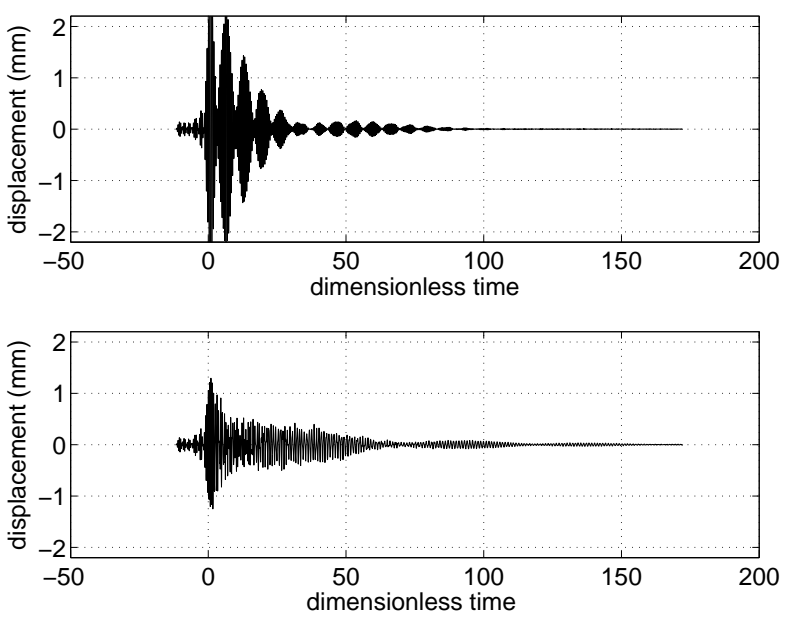

FIGURE 11. TIME DOMAIN OBSERVATION $t / t_{0} \mapsto v(t)$ RELATED TO THE LINEAR (UPPER GRAPH) AND THE NONLINEAR (LOWER GRAPH) CASES FOR $\mathbb{B}_{e}^{2}=[1.78,2.34]$ and $f_{0}=$ $2.75 N$.

\section{NONLINEAR SENSITIVITY ANALYSIS ACCORDING TO THE TYPE OF UNCERTAINTIES}

In the present case, the mean NL-ROM is constructed by modal analysis without substructuring techniques. Thus, the uncertainties are not considered as independent from one blade to 

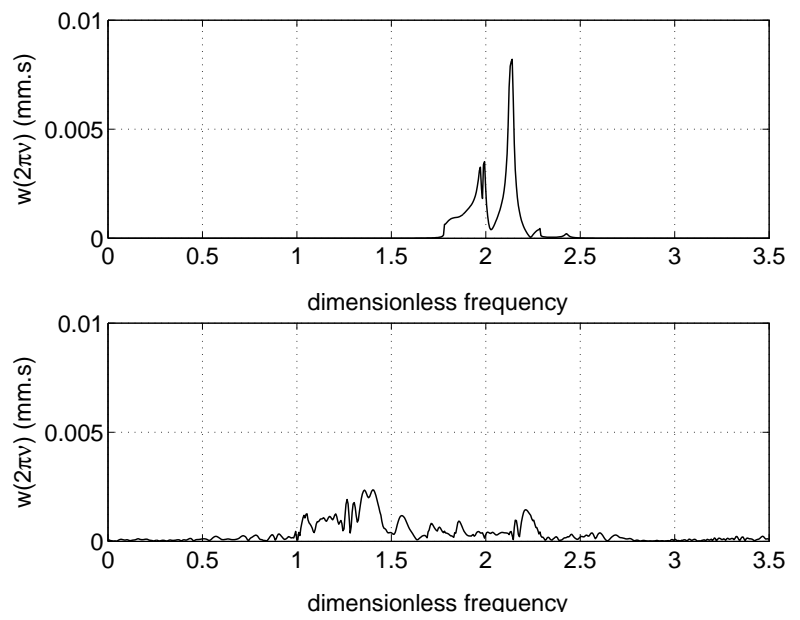

FIGURE 12. FREQUENCY DOMAIN OBSERVATION $v / v_{0} \mapsto$ $w(2 \pi v)$ RELATED TO THE LINEAR (UPPER GRAPH) AND THE NONLINEAR (LOWER GRAPH) CASES FOR $\mathbb{B}_{e}^{2}=[1.78,2.34]$ and $f_{0}=2.75 N$.

another one, which is coherent with the structure under consideration belonging to the class of integrated bladed disks, which are manufactured from a unique solid piece of material. In the present analysis, for a better understanding of the phenomenon, only the nonlinear part of the operators are considered to be deterministic. The mistuning level is thus controlled by the $\mathbb{R}^{5}$ vector $\delta=\left(\delta_{M}, \delta_{D}, \delta_{C}, \delta_{K_{c}}, \delta_{K}\right)$.

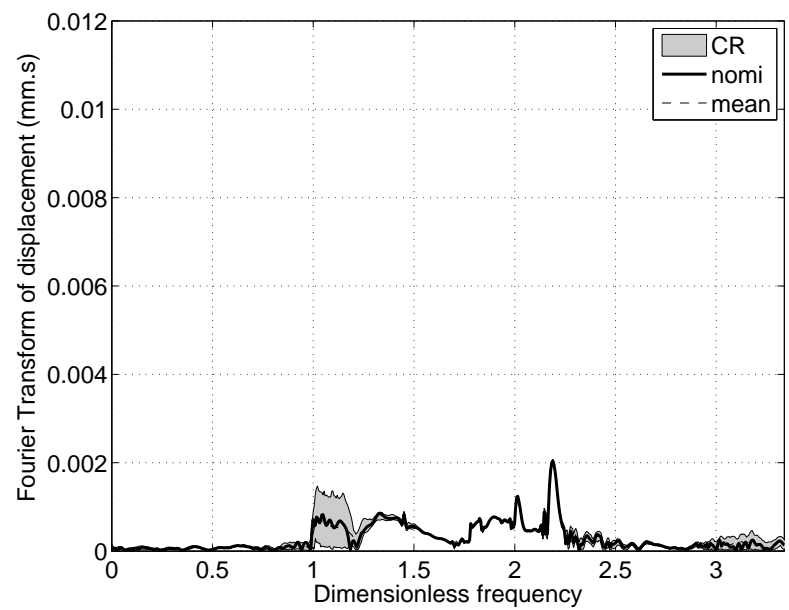

FIGURE 13. STOCHASTIC ANALYSIS: FREQUENCY DOMAIN OBSERVATION RELATED TO THE NONLINEAR CASE WHEN $\delta_{C}=0.1:$ MEAN MODEL (THICK LINE), MEAN OF THE STOCHASTIC MODEL (THIN DASHED LINE), CONFIDENCE REGION (GRAY REGION). EXCITATION FREQUENCY BAND $\mathbb{B}_{e}^{2}$.

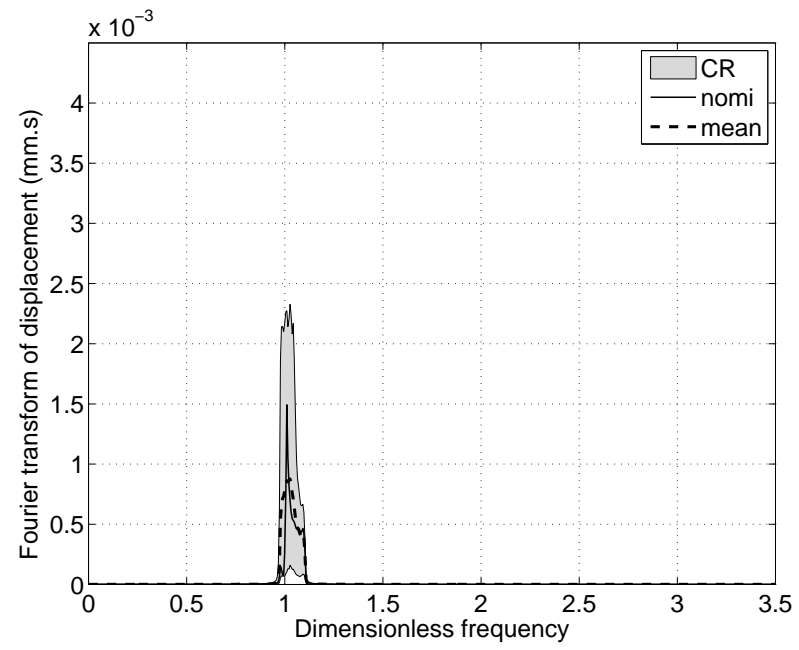

FIGURE 14. STOCHASTIC ANALYSIS: FREQUENCY DOMAIN OBSERVATION RELATED TO THE LINEAR CASE WHEN $\delta_{M}=$ 0.1: MEAN MODEL (THICK LINE), MEAN OF THE STOCHASTIC MODEL (THIN DASHED LINE), CONFIDENCE REGION (GRAY REGION). EXCITATION FREQUENCY BAND $\mathbb{B}_{e}^{1}$

The mistuning analysis is carried out in the frequency domain, which then requires a Fourier transform of the observation. First, a sensitivity analysis is conducted in order to identify the dispersion parameters yielding the most significant effects on the mistuned response represented by the random variable $W(2 \pi v)$ similar to observation $w(2 \pi v)$ in the tuned case.

As expected for the linear mistuned case, it can be observed that an uncertainty level of 0.1 for the gyroscopic coupling, or/and the centrifugal stiffness or/and the dissipation terms, has a very limited impact on the linear mistuned response, whether the excitation band is $\mathbb{B}_{e}^{1}$ or $\mathbb{B}_{e}^{2}$. The confidence region of observations $W^{N L}(2 \pi v)$ with a probability level set to 0.95 has been compared, when the frequency excitation band is $\mathbb{B}_{e}^{1}$ or $\mathbb{B}_{e}^{2}$. In the first case, it can be shown that the nonlinear response seems to be insensitive to uncertainties on the whole band of analysis $\mathbb{B}$. In the second case, Fig. 13 shows that the nonlinear mistuned behavior is substantially different. On one side, the nonlinear mistuned response behaves almost like its tuned counterpart in the part of the frequency band corresponding to the frequency band of excitation $\mathbb{B}_{e}^{2}$. On the other side, the uncertainties spread throughout the geometrical nonlinearities, yielding large confidence regions for the nonlinear mistuned response in $\mathbb{B} \backslash \mathbb{B}_{e}^{2}$, more particularly in the dimensionless sub-frequency range $\mathbb{B}_{s}=[1,1.2]$. The dynamical analysis in $\mathbb{B}_{s}$ shows realizations with amplification levels around 2 whereas the mistuned response remains almost unchanged in $\mathbb{B}_{e}^{2}$. Note that a similar nonlinear mistuned behavior can be observed for the cases of uncertain centrifugal stiffness or uncertain dissipation. 
Figures 14 and 16 (resp. 15 and 17) displays the graphs of the confidence region of observations $W^{L}(2 \pi v)$ and $W^{N L}(2 \pi v)$ with a probability level set to 0.95 for a mass uncertainty level $\delta_{M}=0.1$ and a load level $f_{0}=2.5 \mathrm{~N}$ in the excitation band $\mathbb{B}_{e}^{1}$ (resp. $\mathbb{B}_{e}^{2}$ ). By comparing Figures 14 and 15, it can be seen for the usual linear mistuned case that the random response of the blade is particularly sensitive to uncertainties yielding consequent amplification response levels. For the case corresponding to an excitation located in $\mathbb{B}_{e}^{1}$, the presence of geometric nonlinearities drastically improves the robustness of the random response in $\mathbb{B}_{e}^{1}$, since the upper confidence region envelope of the confidence region does not exceed the tuned resonance. Moreover, the response level outside $\mathbb{B}_{e}^{1}$ is one order of magnitude smaller, and the presence of uncertainties does not affect the nonlinear dynamical behaviour of the blades.

By comparing Figures 16 and 17, it can be seen again that the presence of geometric nonlinearities tends to inhibit the nonlinear dynamical amplification induced by the mistuning in the frequency band $\mathbb{B}_{e}^{2}$ corresponding to the excitation frequency band, limiting the dynamical amplification level to a magnitude 1.2. Moreover, a widespread of uncertainties is observed in $\mathbb{B} \backslash \mathbb{B}_{e}^{2}$ with dynamic amplification levels of magnitude 2.5 when performing the nonlinear dynamic analysis in subfrequency band $\mathbb{B}_{\text {sub }}=[1,1.6]$. In this band, one then can see nonlinear mistuned response levels similar to the nonlinear tuned response located in $\mathbb{B}_{e}^{2}$. Note that a similar behavior is observed for the case of elastic stiffness uncertainties.

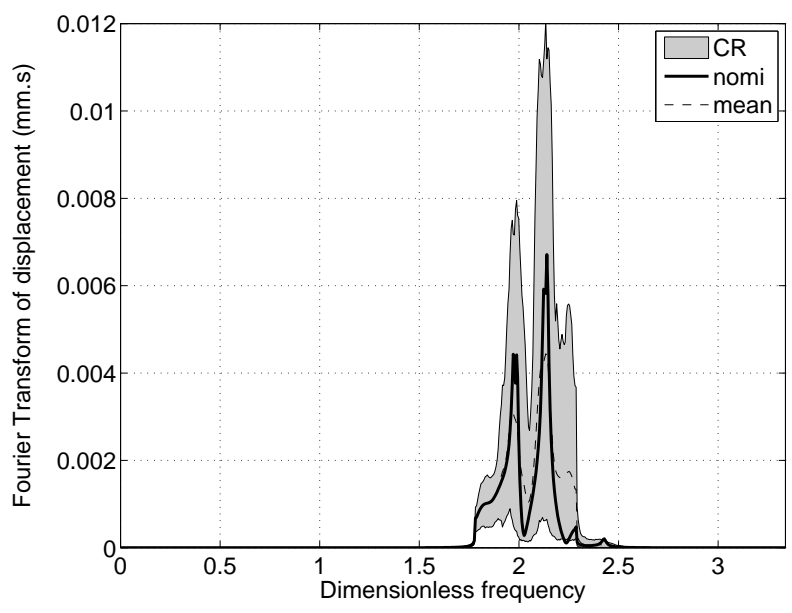

FIGURE 15. STOCHASTIC ANALYSIS: FREQUENCY DOMAIN OBSERVATION RELATED TO THE LINEAR CASE WHEN $\delta_{M}=$ 0.1: MEAN MODEL (THICK LINE), MEAN OF THE STOCHASTIC MODEL (THIN DASHED LINE), CONFIDENCE REGION (GRAY REGION). EXCITATION FREQUENCY BAND $\mathbb{B}_{e}^{2}$

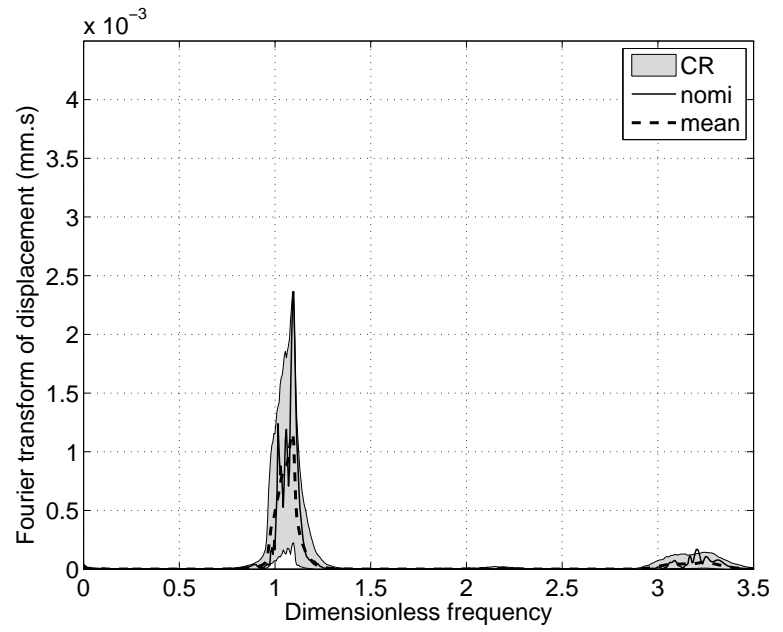

FIGURE 16. STOCHASTIC ANALYSIS: FREQUENCY DOMAIN OBSERVATION RELATED TO THE NONLINEAR CASE WHEN $\delta_{M}=0.1:$ MEAN MODEL (THICK LINE), MEAN OF THE STOCHASTIC MODEL (THIN DASHED LINE), CONFIDENCE REGION (GRAY REGION). EXCITATION FREQUENCY BAND $\mathbb{B}_{e}^{1}$

\section{NONLINEAR DYNAMICAL ANALYSIS OF THE MIS- TUNED RESPONSE}

From now on, the analysis is focused on the excitation frequency band $\mathbb{B}_{e}^{2}$, which exhibits the complex dynamic situation described above. The load level is fixed to $f_{0}=2.5 \mathrm{~N}$ and the uncertainty level is set to $\delta=\left(\delta_{M}, \delta_{D}, \delta_{C}, \delta_{K_{c}}, \delta_{K}\right)=$

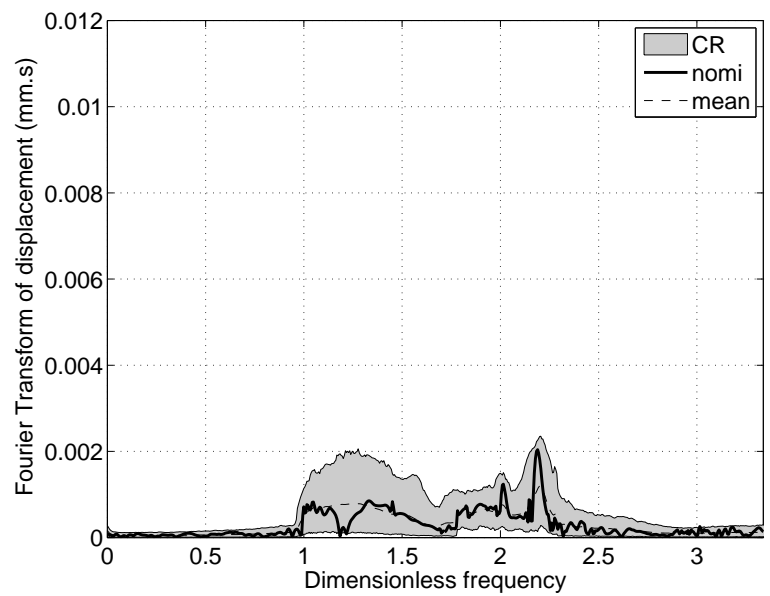

FIGURE 17. STOCHASTIC ANALYSIS: FREQUENCY DOMAIN OBSERVATION RELATED TO THE NONLINEAR CASE WHEN $\delta_{M}=0.1$ : MEAN MODEL (THICK LINE), MEAN OF THE STOCHASTIC MODEL (THIN DASHED LINE), CONFIDENCE REGION (GRAY REGION). EXCITATION FREQUENCY BAND $\mathbb{B}_{e}^{2}$ 
$(\delta, 0.2,0.2,0.2, \delta)$. Thus, the effects of mass and elastic uncertainties combined to the uncertainties issued from the rotational effects are taken into account in the analysis. A parametric analysis according parameter $\delta$ is carried out in order to establish a comparison of the mistuning effects between the linear and the nonlinear mistuned cases. For fixed $v / v_{0} \in$ $\mathbb{B}$, let $Y(2 \pi v)$ be the random dynamic observation defined by $Y(2 \pi v)=\frac{W(2 \pi v)}{\max _{v / v_{0} \in \mathbb{B}} w(2 \pi v)}$. Figures 18 and 19 show the confidence region of the linear and nonlinear observations $Y^{L}(2 \pi v)$ and $Y^{N L}(2 \pi v)$ when $\delta=0.16$. In frequency band $\mathbb{B}_{e}^{2}$, it can be observed that an increase of the mistuning yields a uniform spread in the frequency domain around the main resonance, yielding a weak robustness with respect to uncertainties. It is also clearly seen that the linearized assumption tends to increase the extreme values of the response levels. The geometric nonlinear effects clearly inhibit the amplification of the random response. More particularly, the extreme values related to $Y^{N L}(2 \pi v)$ yield moderate amplification even if the confidence region remains relatively broad.

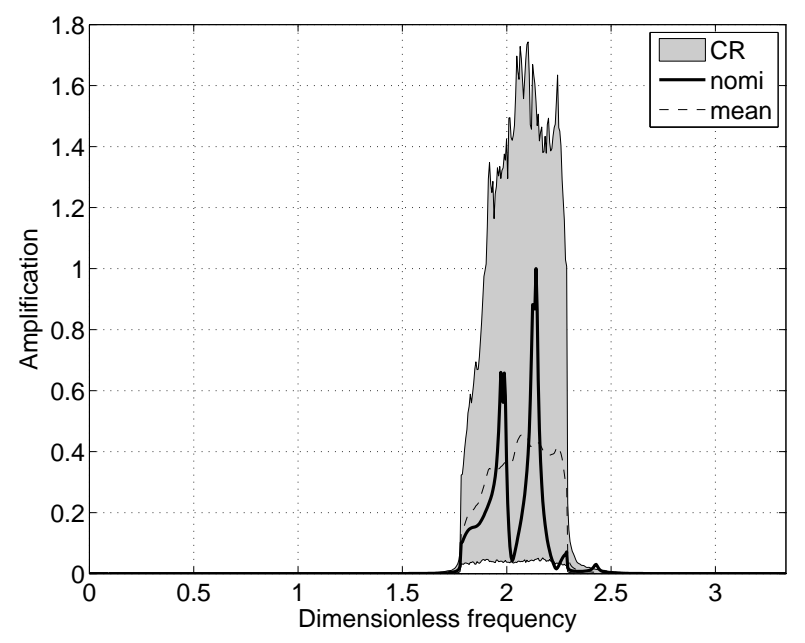

FIGURE 18. STOCHASTIC ANALYSIS: FREQUENCY DOMAIN OBSERVATION RELATED TO THE LINEAR CASE $Y^{L}(2 \pi v)$ when $\delta_{K}=\delta_{M}=0.16$ AND $\delta_{K_{c}}=\delta_{C}=\delta_{D}=0.2$ : MEAN MODEL (THICK LINE), MEAN OF THE STOCHASTIC MODEL (THIN DASHED LINE), CONFIDENCE REGION (GRAY REGION).

\section{CONCLUSION}

A novel methodology adapted for the mistuning analysis of bladed disks in the context of high loads inducing strong geometric nonlinear effects has been applied to an industrial bladed

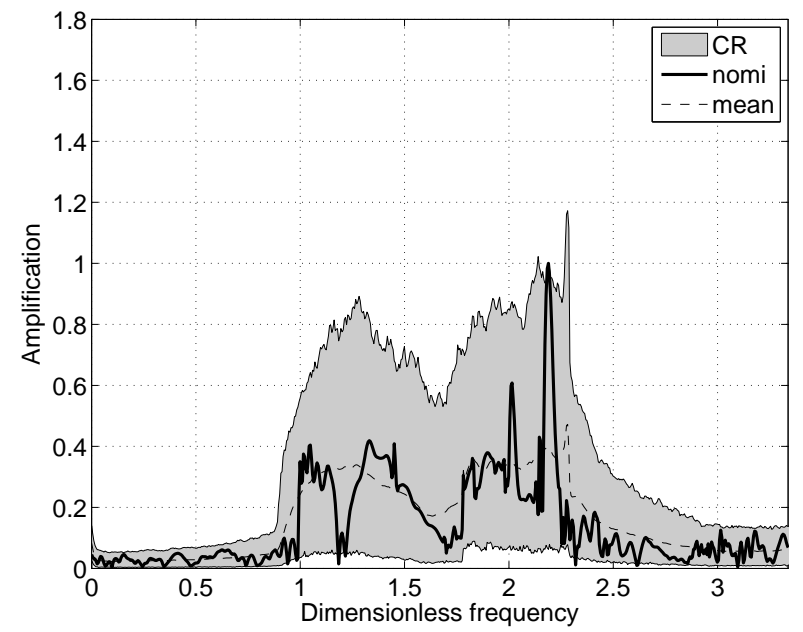

FIGURE 19. STOCHASTIC ANALYSIS: FREQUENCY DOMAIN OBSERVATION $Y^{N L}(2 \pi v)$ RELATED TO THE NONLINEAR CASE WHEN $\delta_{K}=\delta_{M}=0.16$ AND $\delta_{K_{c}}=\delta_{C}=\delta_{D}=0.2$ : MEAN MODEL (THICK LINE), MEAN OF THE STOCHASTIC MODEL (THIN DASHED LINE), CONFIDENCE REGION (GRAY REGION).

disk. The main goal of the approach is to quantify those geometric nonlinear effects on the dynamical response of the mistuned structure. On this basis, two load cases have been investigated, corresponding to situations which are known to yield moderate and drastic dynamical amplifications in the usual linear mistuned case. Numerical results display new complex dynamical behaviors of the dynamical response of the blades.

For an excitation corresponding to pure blade modes, in the very low-frequency range, the nonlinear tuned response exhibits higher amplitude levels, while mainly remaining located in the frequency band of excitation. On the opposite, when the excitation is located around a veering, characterizing a strong coupling between the blade modes and the disk modes, a sudden stiffening of the vibration motion is observed and the nonlinear tuned response is spread outside the frequency band of excitation, yielding secondary resonances corresponding to sub-harmonics and whose contribution cannot be longer neglected. In this case, a sensitivity analysis with respect to the load level exhibits in this case a load threshold, for which an irregular behavior is observed.

Considering the nonlinear mistuned case, it is seen that the geometric nonlinear effects acts like an opportune ground for the propagation of uncertainties. In particular, the robustness of the random response remains strong in the frequency band of excitation, yielding reasonable amplification levels. Nevertheless, such robustness suddenly falls in the sub-harmonic range giving rise to consequent local amplification levels. 


\section{ACKNOWLEDGMENT}

This work was supported by the DGA (French defence procurement agency) in the context of the TURBODYNA project (project number ANR-13-ASTR-0008-01) related to the ANR ASTRID research program (specific support scheme for research works and innovation defence).

\section{REFERENCES}

[1] Wei, S.-T., Pierre, C., 1988. "Localization Phenomena in Mistuned Assemblies with Cyclic Symmetry Part II: Forced Vibrations", ASME Journal of Vibration, Acoustics Stress and Reliability in Design, 110(4), pp. 439-449.

[2] Bladh, R., Castanier, M.-P., Pierre,C., 2001. "Componentmode-based reduced-order modeling techniques for mistuned bladed disks - Part I: Theoretical models, ASME Journal of Engineering Gas, Turbine and Power, 123(1), pp. 89-99.

[3] Capiez-Lernout, E., Soize, C., 2004. 'Nonparametric modeling of random uncertainties for dynamic response of mistuned bladed disks", ASME Journal of Engineering for Gas, Turbine and Power, 126(3), pp. 610-618.

[4] Mbaye, M., Soize, C., Ousty, J.-P., 2010. "A reduced-order model of detuned cyclic dynamical systems with geometric modifications using a basis of cyclic modes", ASME Journal of Engineering for Gas Turbines and Power, 132(11), paper 112502.

[5] Rivas-Guerra, A.-J., Mignolet, M.-P., Delor, J.-P., 2001. "Identification of mistuning characteristics of bladed disks from free response data - Part II", ASME Journal of Engineering Gas, Turbine and Power, 123(2), pp. 404-411.

[6] Castanier, M.P., Pierre, C., 2002. "Using intentional mistuning in the design of turbomachinery rotors", AIAA Journal, 40(10), pp. 2077-2086.

[7] Choi, B.-K., Lentz, J., Rivas-Guerra A.J., Mignolet, M.-P., 2003. "Optimization of intentional mistuning patterns for the reduction of the forced response effects of unintentional mistuning: Formulation and assessment", ASME Journal of Engineering for Gas Turbines and Power, 125(1), pp. 131140.

[8] Mbaye, M., Soize, C., Ousty, J.-P., Capiez-Lernout, E., 2013. "Robust Analysis of Design in Vibration of Turbomachines", ASME Journal of Turbomachinery, 135(2), paper 021008.

[9] Laxalde, D., Thouverez, F., Sinou, J.-J., Lombard, J.-P., 2007. "Qualitative analysis of forced response of blisks with friction ring dampers", European Journal of Mechanics, A/Solids, 26(4), pp. 676-687.

[10] Petrov, E.-P., 2012., "Analysis of flutter-induced limit cycle oscillations in gas-turbine structures with friction, gap, and other nonlinear contact interfaces", ASME Journal of Turbomachinery, 134(6), paper 061018.
[11] Szwedowicz, J., Secall-Wimmel, T. and Dünck-Kerst, P., 2008., "Damping Performance of Axial Turbine Stages With Loosely Assembled Friction Bolts; The Non-Linear Dynamic Assessment", ASME Journal of Engineering for Gas Turbines and Power, 130(3), paper 032505.

[12] Muryavov, A.-A., Rizzi, S.-A., 2003., 'Determination of nonlinear stiffness with application to random vibration of geometrically nonlinear structures", Computers and Structures, 81, pp. 1513-1523.

[13] Mignolet, M.-P., Soize,C., 2008., "Stochastic reducedorder models for uncertain geometrically nonlinear dynamical systems",Computer Methods in Applied Mechanics and Engineering, 197, pp. 3951-3963.

[14] Vakakis, A.-F., 1992., "Dynamics of a nonlinear periodic structure with cyclic symmetry", Acta Mechanica, 95(1-4), pp. 197-226.

[15] Grolet, A., Thouverez, F., 2012., "Free and forced vibration analysis of a nonlinear system with cyclic symmetry: Application to a simplified model", Journal of Sound and Vibration, 331(12), pp. 2911-2928.

[16] Capiez-Lernout, E., Soize, C., Mbaye, M., 2014., "Computational Geometrically Nonlinear Vibration Analysis Of Uncertain Mistuned Bladed Disks", paper GT2014-25072, Proceedings of the ASME Turbo Expo 2014: Turbine Technical Conference and Exposition GT2014, June 16-20, 2014, Düsseldorf, Germany.

[17] Soize, C., 1982.,'Medium frequency linear vibrations of anisotropic elastic structures", La recherche aérospatiale (english version), 5, pp. 65-87.

[18] Mignolet, M.-P., Przekop, A., Rizzi, S.A., Spottswood,M.S., 2013., "A review of indirect/nonintrusive reduced-order modeling of nonlinear geometric structures", Journal of Sound and Vibration, 332(10), pp. 2437-2460.

[19] Capiez-Lernout, E., Soize, C., Mignolet, M.-P., 2012., "Computational stochastic statics of an uncertain curved structure with geometrical nonlinearity in threedimensional elasticity", Computational Mechanics, 49(1), pp. 87-97.

[20] Capiez-Lernout, E., Soize, C., Mignolet M.-P., 2014., "Post-buckling nonlinear static and dynamical analyses of uncertain cylindrical shells and experimental validation", Computer Methods in Applied Mechanics and Engineering, 271, pp. 210-230.

[21] Soize, C., 2012., Stochastic Models of Uncertainties in Computational Mechanics, Lecture Notes in Engineering Mechanics 2, American Society of Civil Engineers (ASCE) 